\title{
Oligodendroglial Tumor
}

National Cancer Institute

\section{Source}

National Cancer Institute. Oligodendroglial Tumor. NCI Thesaurus. Code C6960.

A neoplasm of the central nervous system composed predominantly of cells

morphologically resembling oligodendroglia. Oligodendrog lial tumors histologically

comprise a continuous spectrum ranging from well-differentiated neoplasms to frankly

malignant tumors. WHO grading system recognizes two malignancy grades: WHO grade

II for well-differentiated tumors (oligodendrog lioma) and WHO grade III for anaplastic

oligodendrog lioma. (Adapted from WHO) 